\title{
Rhipicephalus appendiculatus ticks transmit Theileria parva from persistently infected cattle in the absence of detectable parasitemia: implications for East Coast fever epidemiology
}

\author{
Cassandra L. Olds ${ }^{1,3}$, Kathleen L. Mason ${ }^{2}$ and Glen A. Scoles ${ }^{2 *}$
}

\begin{abstract}
Background: East Coast fever (ECF) is a devastating disease of cattle and a significant constraint to improvement of livestock production in sub-Saharan Africa. The protozoan parasite causing ECF, Theileria parva, undergoes obligate sexual stage development in its tick vector Rhipicephalus appendiculatus. Tick-borne acquisition and transmission occurs transstadially; larval and nymphal ticks acquire infection while feeding and transmit to cattle when they feed after molting to the next stage. Much of the current knowledge relating to tick-borne acquisition and transmission of T. parva has been derived from studies performed during acute infections where parasitemia is high. In contrast, tick-borne transmission during the low-level persistent infections characteristic of endemic transmission cycles is rarely studied.
\end{abstract}

Methods: Cattle were infected with one of two stocks of T. parva (Muguga or Marikebuni). Four months postinfection when parasites were no longer detectable in peripheral blood by PCR, 500 R. appendiculatus nymphs were fed to repletion on each of the cattle. After they molted to the adult stage, 20 or 200 ticks, respectively, were fed on two naïve cattle for each of the parasite stocks. After adult ticks fed to repletion, cattle were tested for T. parva infection by nested PCR and dot blot hybridization.

Results: Once they had molted to adults the ticks that had fed as nymphs on Muguga and Marikebuni infected cattle successfully transmitted Theileria parva to all naïve cattle, even though T. parva infection was not detectable by nested PCR on salivary gland genomic DNA of a sample of individual ticks. However, a salivary gland homogenate from a single Marikebuni infected tick was able to infect primary bovine lymphocytes. Infection was detected by nested P104 PCR in 3 of 4 calves and detected in all 4 calves by T. parva 18S nested PCR/dot blot hybridization.

Conclusion: We show that $R$. appendiculatus ticks are able to acquire T. parva parasites from infected cattle even in the absence of detectable parasitemia. Although infection was undetectable in a sample of individual ticks, cumulatively as few as 20 ticks were able to transmit $T$. parva to naïve cattle. These results have important implications for our understanding of T. parva transmission by R. appendiculatus in ECF endemic regions.

Keywords: Theileria parva, Rhipicephalus appendiculatus, East Coast fever, Reservoir competence, Persistent transmission, Endemic stability

\footnotetext{
*Correspondence: glen.scoles@ars.usda.gov; gscoles@wsu.edu

${ }^{2}$ USDA, ARS, Animal Disease Research Unit, Washington State University,

Pullman, WA, USA

Full list of author information is available at the end of the article
} 


\section{Background}

Acute infection of cattle with Theileria parva causes a severe lympho-proliferative disease known as East Coast fever (ECF), one of the most significant tick-borne diseases of cattle in Africa [1-3]. Theileria parva most likely co-evolved with African buffalo (Syncerus caffer) as its vertebrate primary host, which display no symptoms of disease when infected [1,3]. Transmission of T. parva to domestic cattle occurs during the feeding of the infected tick vector, Rhipicephalus appendiculatus. Theileria parva sporozoites are released, together with saliva, from tick salivary glands into the feeding site where they infect bovine lymphocytes and develop into the schizont stage, transforming the cells leading to a clonal expansion of schizont-infected lymphocytes. In some of the schizont infected lymphocytes the process of merogony occurs, resulting in the release of merozoites which invade erythrocytes, forming the tick infective piroplasm stage [1,3-7]. The persistent infectious state is thought to be maintained through the slow continuous proliferation of schizont-infected cells, although events governing this process, or where the schizont infected cells reside are poorly understood [1].

The existence of a carrier state for T. parva was first described by Young et al. [8] in 1986, who stressed that persistent infection of cattle was likely "of great importance in maintenance of T. parva infection in the field". Despite this, most of what is known about the tick-borne acquisition and transmission of T. parva by $R$. appendiculatus has been gathered through laboratory studies of cattle undergoing the short-lived acute stage of infection [9-14]. Animals that recover from acute disease can remain persistently infected and serve as reservoirs for $R$. appendiculatus ticks [15]. In areas where natural transmission occurs, a phenomenon known as endemic stability has been described as "a climax relationship between host, agent, vector and environment in which all coexist with the virtual absence of clinical disease" [1]. Under conditions of endemic stability only calves are seen to undergo acute infections and the resulting carrier state may last a number of years [1]. Recently the concept of endemic stability for $T$. parva has been broadened to include the concept that protection may be correlated with closely related avirulent Theileria species circulating naturally in the cattle population [16]. Endemic stability can now be seen as a product of the environment that also includes ecosystem services provided by the broader microbiome [16]. Even under this broader concept of endemic stability, it is important to understand the contribution to herd immunity made by persistent $T$. parva infections, whether naturally acquired or artificially induced through vaccination with live sporozoites.
Acute disease and mortality occur most frequently in areas where endemic stability is absent or has been disrupted $[1,17]$. This could be due to the movement of naive cattle from non-endemic to endemic zones, or the breakdown of tick control, which may have been reducing transmission pressures, limiting the opportunity for early infection of calves and subsequent development of a broad immune response. Endemic stability can also be disrupted by herd improvement programs that introduce more productive European cattle breeds, known to be more susceptible to infection, or by the introduction of virulent $T$. parva strains directly from African buffalo reservoirs at the interface between domestic cattle and wildlife [1, 17-19].

The only currently available method of immunization against acute disease caused by $T$. parva is the Infection and Treatment Method (ITM) of vaccination. Using ITM, cattle are infected with live, cryopreserved infectious sporozoites (which may be derived from multiple T. parva stocks), and immediately treated with longacting tetracycline to attenuate the infection. This method of vaccination has been shown to result in lifelong protection against acute disease, although breakthroughs have been reported to occur [20, 21]. The Muguga stock of T. parva is one component of the ITM cocktail [22], and as the most widely used laboratory stock of $T$. parva it has formed the basis for much of our understanding of immune responses to T. parva and ECF. It has been suggested that the Muguga stock does not induce a carrier state and is cleared from the body one to three months after infection. It has also been suggested that this stock is not tick transmissible after the acute stage of infection has passed [1, 23-26]; however, there is no way of knowing from these previous studies if the parasite is truly absent, or simply undetectable due to lower sensitivity of the assays available at the time. The Marikebuni laboratory stock [27, 28] is widely accepted as inducing a carrier state [23, 29] with circulating parasite DNA detectable in the cattle host for over 400 days after infection [24], remaining tick transmissible for seven months after inoculation [29]. In order to better understand the natural transmission dynamics that cattle might experience in the field we investigated the ability of $R$. appendiculatus ticks to transmit either the Muguga or the Marikebuni stock parasites to naïve cattle after feeding on persistently infected animals with no detectable parasitemia.

\section{Methods}

\section{Tick and parasite stocks}

Rhipicephalus appendiculatus ticks (Muguga colony) and T. parva stocks (Muguga and Marikebuni) were obtained from Dr. Ivan Morrison (Roslin Institute, Edinburgh, Scotland). The tick colony was originally 
collected in Kenya but had been maintained at the Roslin institute on cattle for $\approx 20$ years. At the Animal Disease Research Unit (ADRU) ticks were reared on male Holstein calves and kept at $26{ }^{\circ} \mathrm{C}, 92 \%$ relative humidity $(\mathrm{RH})$ and a 14.5/9.5 h light/dark photocycle for molting and hardening, then moved to $15{ }^{\circ} \mathrm{C}, 92.5 \% \mathrm{RH}$ and a 14.5/9.5 h light/dark photocycle for longer term holding. Ticks infected with $T$. parva were maintained continuously at $26^{\circ} \mathrm{C}$, as above.

\section{Infection and treatment of calves with T. parva Muguga and Marikebuni}

Two male Holstein calves 3 months-old were infected with cryopreserved T. parva sporozoites by needle inoculation above the left scapular lymph node and co-treated with 20 $\mathrm{mg} / \mathrm{kg}$ long acting oxytetracycline (Bio-mycin 200, Boehringer Ingelheim, Ingelheim am Rhein, Germany). Calf C-1448 was infected with Muguga stabilate P2014/2 produced from the salivary glands of 10 ticks (10 tick equivalents), determined by quantitative p104 PCR [30] to contain $1 \times 10^{2} \mathrm{~T}$. parva sporozoites. Calf C-1445 was infected with 10 tick equivalents of Marikebuni stock ED64, determined by quantitative p104 PCR [30] to contain $3 \times 10^{4} \mathrm{~T}$. parva sporozoites. To track infection, daily rectal temperatures were recorded from the day of inoculation until day 21 post-infection. Daily blood samples were taken from the jugular vein on day 0 and on days 5-25 post-infection and thereafter at weekly intervals for the duration of the study.

\section{Monitoring T. parva infection of C-1445 and C-1448, DNA extraction and PCR}

To monitor the presence of T. parva in C-1445 and C1448 , genomic DNA was extracted from $200 \mu$ of whole blood and eluted into $50 \mu \mathrm{l}$ of elution buffer. All DNA extractions were performed using the DNEasy Blood and Tissue kit (QIAGEN GmbH, Hilden, Germany). Unless otherwise stated, PCR reactions were carried out in $32 \mu \mathrm{l}$ volumes using $2 \mu \mathrm{l}$ of template genomic DNA, $1.0 \mu \mathrm{M}$ forward primer, $1.0 \mu \mathrm{M}$ reverse primer, $200 \mu \mathrm{M}$ of dATP, dCTP, dGTP, dTTP, $1.3 \mathrm{U}$ taq, $2.0 \mathrm{mM} \mathrm{MgCl}_{2}$ in 10x reaction buffer (FastStart PCR Polymerase dNTPack kit, Roche, Basel, Switzerland). For nested PCR reactions, the second round of PCR used $0.1 \mu \mathrm{l}$ of the first PCR reaction together with $1.0 \mu \mathrm{M}$ forward primer, $1.0 \mu \mathrm{M}$ reverse primer, $200 \mu \mathrm{M}$ of dATP, dCTP, dGTP, dTTP, $1.25 \mathrm{U}$ taq, $2.0 \mathrm{mM} \mathrm{MgCl}_{2}$ in $10 \times$ reaction buffer and 1X RediLoad (Invitrogen, Carlsbad, USA). Reactions were set up in 8 well strips, with the 8th well of every strip serving as a no template control.

Successful DNA extraction was confirmed by PCR detection of genomic bovine DNA using bovine cytochrome $b$ gene as a target. Cytb1 (5'-CCA TGA GGA CAA ATA TCA TTC TG-3') and Cytb3 (5'-GGG TGT TCD ACT GGY TGB CCY CC-3') primers with an annealing temperature of $55{ }^{\circ} \mathrm{C}$ amplified a $600 \mathrm{bp}$ product [31]. Theileria parva DNA was detected using a nested PCR targeting the p104 gene [24, 26]. External primers IL3231 (5'-ATT TAA GGA ACC TGA CGT GAC TGC-3') and IL755 (5'-TAA GAT GCC GAC TAT TAA TGA CAC C-3') [24] were used with an annealing temperature of $60{ }^{\circ} \mathrm{C}$, amplifying an 827 bp product. Internal forward p104 primer (5'-GGC CAA GGT CTC CTT CAG ATT ACG-3') and reverse primer (5'-TGG GTG TGT TTC CTC GTC ATC TGC-3') [26] were used with an annealing temperature of $55^{\circ} \mathrm{C}$ to amplify an internal product of $277 \mathrm{bp}$.

All PCR reactions were run on a Bio Rad C1000 Touch $^{\text {TM }}$ Thermal Cycler with an initial pre-melt step of $94{ }^{\circ} \mathrm{C}$ for $4 \mathrm{~min} 30 \mathrm{sec}$ followed by 35 cycles of $94{ }^{\circ} \mathrm{C}$ for $30 \mathrm{sec}$, primer specific annealing temperature for $30 \mathrm{sec}$ and extension at $72{ }^{\circ} \mathrm{C}$ for 30 seconds. A final extension step at $72{ }^{\circ} \mathrm{C}$ for $4 \mathrm{~min} 30 \mathrm{sec}$ completed the reaction. PCR products were visualized by $2 \%$ agarose gel electrophoresis with 1:10,000 SYBR safe (Invitrogen, Carlsbad, USA).

\section{Rhipicephalus appendiculatus acquisition of $T$. parva from C-1445 and C-1448}

Rhipicephalus appendiculatus acquisition of T. parva from C-1445 and C-1448 was attempted four months after infection. Five-hundred nymph ticks were applied in tick feeding patches secured to the back of each calf. During the tick-feeding period, daily jugular vein blood samples were drawn and whole blood samples were tested for the presence of $T$. parva DNA as described above. Ticks were allowed to feed to repletion after which they were collected from the feeding patch and kept in incubators to molt into adult stage ticks.

Theileria parva infection rates in the salivary glands of adult ticks, fed as nymphs on C-1445 and C-1448, were measured using nested p104 PCR. To stimulate sporozoite maturation in tick salivary glands 25 male and 25 female adult ticks were fed on the ears of rabbits for four days [32]. After feeding on rabbits, adult ticks were dissected and salivary glands from individual ticks were placed into $100 \mu \mathrm{l}$ of RPMI 1640 media supplemented with $10 \%$ heat-inactivated fetal calf serum, $2 \mathrm{mM}$ L-glutamine, 100 units $/ \mathrm{ml}$ penicillin, $50 \mu \mathrm{g} / \mathrm{ml}$ streptomycin and $5 \times 10^{-5} \mathrm{M} \beta$ mercaptoethanol (cRPMI). Salivary glands were homogenized with a sterile micropestle in a $1.5 \mathrm{ml}$ microcentrifuge tube. Half the homogenate was used to infect bovine peripheral blood mononuclear cells (PBMC) and the remainder stored at $-20{ }^{\circ} \mathrm{C}$ until genomic DNA was extracted and tested by nPCR for the presence of $T$. parva DNA. 


\section{Infection of PBMC with $T$. parva from tick salivary gland homogenate}

Theileria parva sporozoites from tick salivary glands are able to infect bovine PBMCs in vitro. To further evaluate the $T$. parva infection in adult ticks, $2 \times 10^{5}$ PBMCs from an uninfected donor calf were combined with $50 \mu \mathrm{l}$ of the tick salivary gland homogenate from each tick. The cells were incubated for one hour at $37{ }^{\circ} \mathrm{C}$ in $5 \%$ $\mathrm{CO}_{2}$. Samples were agitated every 15 minutes to resuspend cells. Following incubation, $1 \mathrm{ml}$ of cRPMI was added to each tube and centrifuged at $500 \times g$ for $3 \mathrm{~min}$ to wash the cells. Pelleted cells were resupended in 100 $\mu \mathrm{l}$ of cRPMI and plated into individual wells of a 96 well plate. Cells were incubated for 3 weeks at $37{ }^{\circ} \mathrm{C}$ in $5 \%$ $\mathrm{CO}_{2}$ after which, cells were harvested and genomic DNA extracted. DNA was eluted into $30 \mu \mathrm{l}$ of elution buffer and kept at $-20{ }^{\circ} \mathrm{C}$ until use. Samples were tested for the presence $T$. parva DNA by nested p104 PCR as described above.

\section{DNA extraction from tick salivary glands and PCR detection of T. parva}

Genomic DNA was extracted from the remaining $50 \mu \mathrm{l}$ of tick salivary gland homogenate using the DNEasy Blood and Tissue kit protocol for extraction of DNA from tissue. DNA was eluted into $30 \mu \mathrm{l}$ of elution buffer. To confirm that DNA was successfully isolated from each salivary gland sample, tick DNA was detected using primers $16 \mathrm{~S}+1$ (5'-CTG CTC AAT GAT TTT TTA AAT TGC TGT GG-3') and 16S-1 (5'-CCG GTC TGA ACT CAG ATC AAG T-3') targeting a 460 bp fragment of the tick mitochondrial 16S rRNA gene [33]. Theileria parva DNA was detected by nested p104 PCR in the same manner as described for bovine blood.

\section{Tick transmission of T. parva from persistently infected to naïve hosts}

To determine if adult ticks fed as nymphs on persistently infected cattle were able to transmit $T$. parva to naïve cattle, ticks were fed on four naïve calves. On calf C-1470, 10 male and 10 female adult ticks from Marikebuni infected calf C-1445 were fed. 100 male and 100 female adult ticks from C-1445 were fed on calf C-1471. On calf C-1472, 10 male and 10 female adult ticks from Muguga infected calf C-1448 were fed while on calf C-1473, 100 male and 100 female adult ticks from C-1448 were fed. Ticks were applied as a group to each calf in a feeding patch and allowed to feed until female ticks detached at repletion. After all the female ticks had naturally detached male ticks were manually removed using forceps. Salivary glands were dissected from all males and replete females; DNA was extracted and tested for the presence of T. parva DNA by nested p104 PCR.
Animals were monitored daily for symptoms of ECF such as fever (rectal temperature above $39.5{ }^{\circ} \mathrm{C}$ ), swollen lymph nodes, loss of appetite and coughing. Peripheral blood was sampled daily to confirm parasite transmission by PCR. Nested p104 PCR was carried out daily on genomic DNA extracted from both whole blood and separated lymphocytes. Because the schizont stage of T. parva proliferates in lymphocytes, PBMCs were isolated daily from $5 \mathrm{ml}$ of blood using ficol-paque gradient centrifugation to increase the sensitivity of parasite detection. DNA was extracted from $5 \times 10^{6}$ PBMCs and eluted into $50 \mathrm{ul}$ of elution buffer. To increase the quantity of DNA tested for each animal, 10 reactions were performed for each lymphocyte sample. For each animal, days 0 and 5-21 post-tick application were tested. In addition to p104 PCR results, PCR targeting the $T$. parva $18 \mathrm{~S}$ rRNA gene (described below) was performed on each sample, $18 \mathrm{~S}$ rRNA PCR products were detected by Southern blotting; positive samples were sequenced for confirmation.

\section{PCR and Southern blot detection of T. parva targeting the $18 \mathrm{~S}$ rRNA gene}

Nested 18S rRNA PCR reactions were performed in triplicate for each sample to allow for sufficient sample material. First round reactions were carried out using forward primer Theileria 18S_F1 (5'-GAG GGA GCC TGA GAA ACG-3') and reverse primer Theileria 18S_R1 (5'-GGT ATC TGA TCG TCT TCG ATC C-3') with an annealing temperature of $65{ }^{\circ} \mathrm{C}$. Second round PCR primers were Bab 18S_437-461 F (5'-AAT CCT GAC ACA GGG AGG TAG TGA C-3') and Bab 18S_898-873 R (5'-CTA AGA ATT TCA CCT CTG ACA GT-3') [34]. The products from three PCR reactions were pooled together and visualized on a Southern dot blot by hybridization with a digoxigenin (DIG)-labeled T. parva probe.

The $T$. parva probe was produced using forward and reverse primers each with a 5' DIG label and to increase the signal strength additional DIG labeled dUTPs added to the PCR mix during synthesis using the PCR DIG Probe Synthesis Kit (Roche, Basel, Switzerland). The template for PCR amplification of the probe was provided by the full length Muguga 18S rRNA gene cloned into pCR4 TOPO vector (Invitrogen, Carlsbad, USA). In a $50 \mu \mathrm{l}$ probe synthesis reaction, $66 \mathrm{pg}$ of template was added to $0.5 \mu \mathrm{M}$ forward (5'-/5DigN/ GCT GCA TCG CTG GTC TCC-3'), and $0.5 \mu \mathrm{M}$ reverse (5'-/5DigN/ CAT CCA GAC AAA GCG AAC TCC-3') primers, $200 \mu \mathrm{M}$ dATP, dCTP, dGTP, $165 \mu \mathrm{M}$ dTTP and $35 \mu \mathrm{M}$ DIG-dUTP in 10× reaction buffer with a final concentration of $75 \mu \mathrm{M} \mathrm{MgCl}$. Probe synthesis reactions were run according to the manufacturer's instructions. After PCR cycling was complete, the DIG-labeled probe was heated to $95{ }^{\circ} \mathrm{C}$ for $5 \mathrm{~min}$ and 


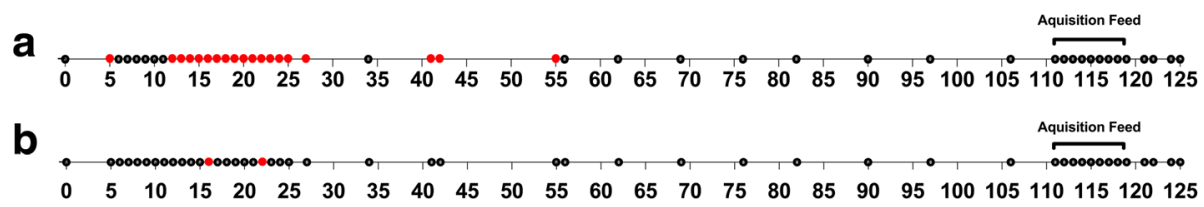

Fig. 1 Detection of T. parva DNA by nested p104 PCR in Marikebuni infected calf C-1445 (a) and Muguga infected calf C-1448 (b). Dates tested post-infection are represented by circles; circles are shown in red on dates where parasite DNA was detected

immediately transferred to ice after which, the product was added to $20 \mathrm{ml}$ of DIG Easy Hyb buffer (Roche, Basel, Switzerland) pre-warmed to $42^{\circ} \mathrm{C}$. The probe was reused for each blot by storing at $-20{ }^{\circ} \mathrm{C}$ between uses. Before each reuse, the probe was heated at $72{ }^{\circ} \mathrm{C}$ for $10 \mathrm{~min}$. Positively charged Magnacharge nylon transfer membrane (GE Osmonics, Minnetonka, USA) was wet in $0.1 \%$ saline-sodium citrate (SSC) buffer ( $15 \mathrm{mM} \mathrm{NaCl}, 1.5 \mathrm{mM}$ sodium citrate, $\mathrm{pH} 7$ ). The membrane was placed onto a 96 well dot blotter, $35 \mu \mathrm{l}$ of combined PCR products was mixed with $100 \mu \mathrm{l} 0.1 \% \mathrm{SSC}$ and heated to $95{ }^{\circ} \mathrm{C}$ for 2 min then immediately transferred to ice. Samples remained on ice until loading into individual wells of a 96 well dot blotter and passed through using vacuum suction. Membranes with the DNA fragments were cross-linked in a UV cross-linker $(1200 \mu$ Joules $\times 100$, UV Stratalinker 2400). Once cross-linked, membranes were sealed within hybridization bags (Roche, Basel, Switzerland) and blocked with $30 \mathrm{ml}$ of DIG blocking buffer (DIG Wash and Block Buffer Set, Roche, Basel, Switzerland) for 30 min with gentle rocking. Following blocking, the membrane was equilibrated in DIG Easy Hyb buffer for 30 min. Hybridization with the probe was carried out at $42{ }^{\circ} \mathrm{C}$ for one hour with rocking. Following hybridization, the membrane was briefly washed in DIG Easy Hyb Wash buffer and then blocked with $30 \mathrm{ml}$ Blocking buffer for $30 \mathrm{~min}$. Following blocking, a 1:20,000 dilution of anti-Digoxigenin-AP Fab fragments (Roche, Basel, Switzerland) in DIG blocking buffer was added and incubated for $30 \mathrm{~min}$ at room temperature with rocking. The membrane is then washed twice in $100 \mathrm{ml}$ DIG washing buffer for $15 \mathrm{~min}$ followed by equilibration in $5 \mathrm{ml}$ of DIG Detection buffer. $20 \mu \mathrm{l}$ of
CDP-Star (Roche, Basel, Switzerland) was added to $2 \mathrm{ml}$ DIG detection buffer, incubated for 5 min before being exposed to Blue Lite Autorad film (GeneMate, Radnor, Pennsylvania) for $10 \mathrm{~s}$.

Where positive Southern blot dots were observed, the remaining PCR product was cleaned using the QIAquick PCR Purification Kit and sequenced using the Bab 18S_437461 primer. Sequences were compared to the T. parva 18s rDNA sequences obtained from GenBank (HQ895984.1) using the Clustal Omega (https://www.ebi.ac.uk/Tools/msa/ clustalo/) multiple sequence alignment tool.

\section{Results}

Infection of calves with T. parva

Inoculation of calves C-1445 and C-1448 with T. parva sporozoites and concurrent treatment oxytetracycline resulted in mild clinical disease characterized by marked enlargement of the lymph node draining the site of sporozoite inoculation. At no point was fever (rectal temperature above $39.5{ }^{\circ} \mathrm{C}$ ) observed and no additional oxytetracycline treatment was required. Parasites were detectable by nested p104 PCR in peripheral blood of the Marikebuni-infected calf C-1445 until day 55 postinfection and until day 22 in the Muguga-infected calf C-1448 (Fig. 1). Beyond this, parasites were not detectable in weekly blood samples from either animal, either before or during tick feeding at 4 months post-infection.

\section{Infection rates in adult ticks fed as nymphs on persistently infected cattle C-1445 and C-1448}

After fed nymphs had molted to the adult stage, genomic DNA was successfully extracted from salivary

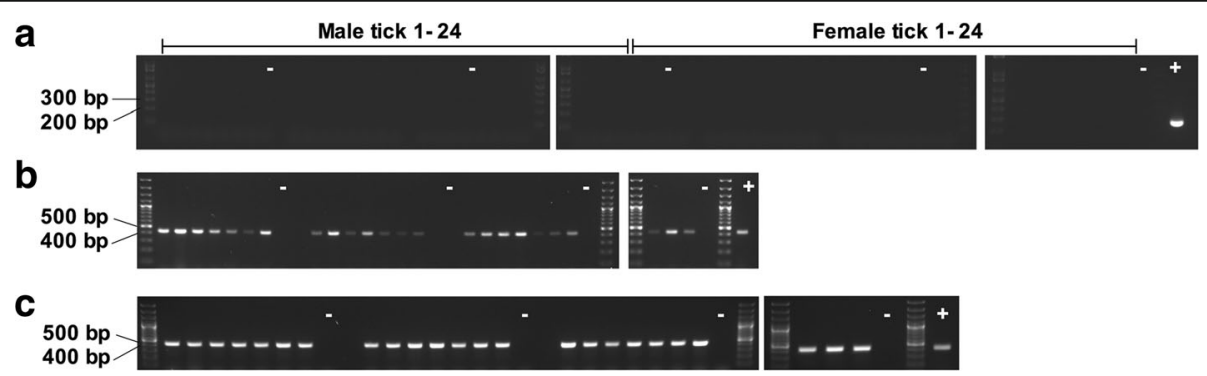

Fig. 2 Determining T. parva infection rate by p104 nested PCR in adult ticks fed as nymphs on Marikebuni infected calf C-1445 (a). Tick 16S PCR confirms genomic DNA was successfully isolated from all male (b) and female (c) ticks 


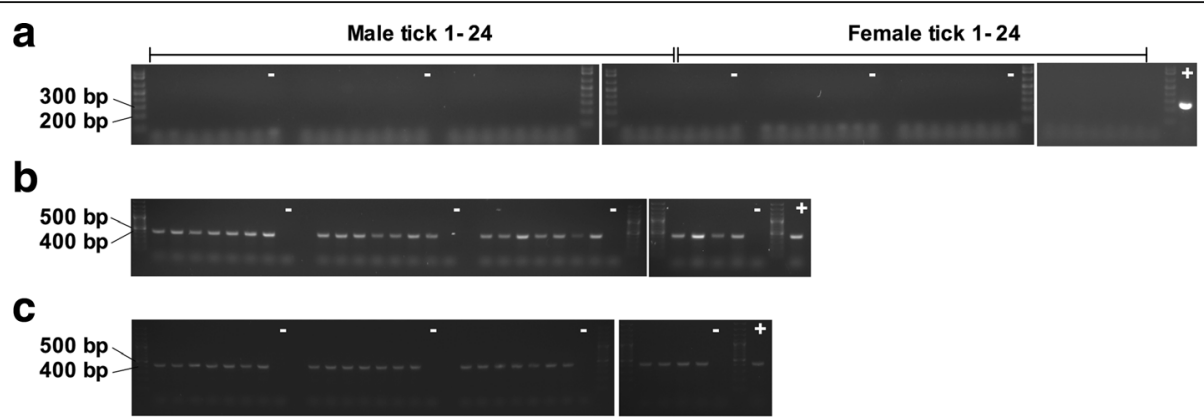

Fig. 3 Determining T. parva infection rate by p104 nested PCR in adult ticks fed as nymphs on Muguga infected calf C-1448 (a). Tick 16S PCR confirms genomic DNA was successfully isolated from all male (b) and female (c) ticks

gland homogenate of ticks fed on Marikebuni-infected calf C-1445 (Fig. 2b, c) and Muguga-infected calf C-1448 (Fig. 3b, c). No T. parva DNA could be detected in any of the 24 male or 24 female ticks analyzed (Figs. 2a and 3a). However, T. parva DNA was detected in lymphocytes incubated with Marikebuni female tick \#17 salivary gland homogenate indicating viable sporozoites were present (Fig. 4).

\section{Transmission of $T$. parva to naïve calves by ticks fed on C-1445 and C-1448 four months post-infection}

To determine if adult ticks that had been fed as nymphs on persistently infected cattle had been able acquire parasite levels sufficient to transmit $T$. parva to naïve calves, 20 (10 male and 10 female) or 200 (100 male and 100 female) ticks were fed, respectively on 2 different susceptible calves for each parasite stock. Adult ticks fed as nymphs on Marikebuni-infected C-1445 transmitted T. parva to naïve cattle C-1470 and C-1471 on which 20 and 200 ticks, respectively were applied. No parasites were detected in whole blood samples; however, using nested p104 PCR, $T$. parva DNA was detected in lymphocytes from C-1470 on day 10 and 12 (Fig. 5a) and on day 10 by $18 \mathrm{~S}$ rRNA gene nPCR and Southern blotting (Fig. 6a). In C-1471 T. parva DNA was detected by p104 nPCR in lymphocytes on day 9, 10 and 11 (Fig. 5b) and on days $8,9,10$ and 12 by $18 \mathrm{~S} \mathrm{nPCR}$ and Southern blotting (Fig. 6b).

Ticks fed on Muguga-infected C-1448 transmitted $T$. parva to C-1472 and C-1473, on which 20 and 200 ticks, respectively were applied. Theileria parva DNA was detected in C-1473 lymphocytes on day 8, 9, 13 and 14 post-tick application using p104 nPCR (Fig. 5d) and on day 10 by $18 \mathrm{~S}$ rRNA gene nPCR and Southern blotting (Fig. 6d). In C-1472, T. parva DNA was detected in lymphocytes by Southern blotting on day 8 and 9 (Fig. 6c) but was not detected using p104 nPCR (Fig. 5c). Sequences of the $18 \mathrm{~S}$ rRNA gene PCR products for all animals were identified as T. parva (Fig. 7). No T. parva DNA could be detected in the salivary glands from replete ticks collected after transmission feeding on each calf using either p104 or 18S rRNA nPCR.

\section{Discussion}

Our current understanding of tick-borne acquisition and transmission of $T$. parva has been derived primarily from studies of tick-pathogen-host interactions occurring during the acute stage of infection in animals undergoing severe ECF. During acute infection, high parasitemia is observed in the bovine host, which translates to quantifiable infection levels in ticks feeding on infected animals. Under natural field conditions, animals undergoing acute disease without treatment may not survive long enough for tick feeding to be completed making them dead-end hosts. Animals that do recover from acute stage infection may remain low-level carriers for many months. Acquisition and transmission of T. parva from these persistently infected cattle is thus more representative of the natural transmission cycle in areas where T. parva is endemic.

We have shown that even though parasites are undetectable in the peripheral blood at the time of tick feeding, ticks can and do acquire the parasite after feeding on cattle suggesting that red blood cells infected

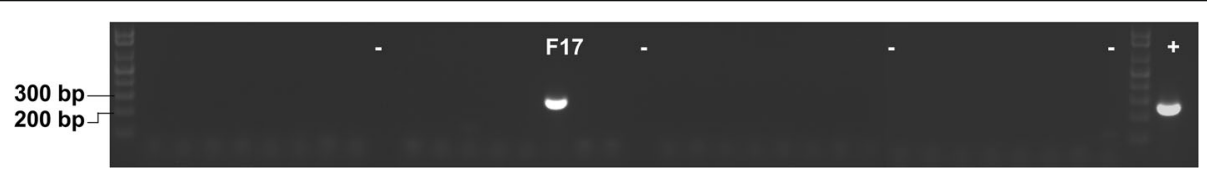

Fig. 4 Detection of T. parva DNA by p104 nested PCR in lymphocyte cultures from Marikebuni-infected C-1445. Theileria parva DNA could only be detected in the lymphocyte culture from Female tick \#17 


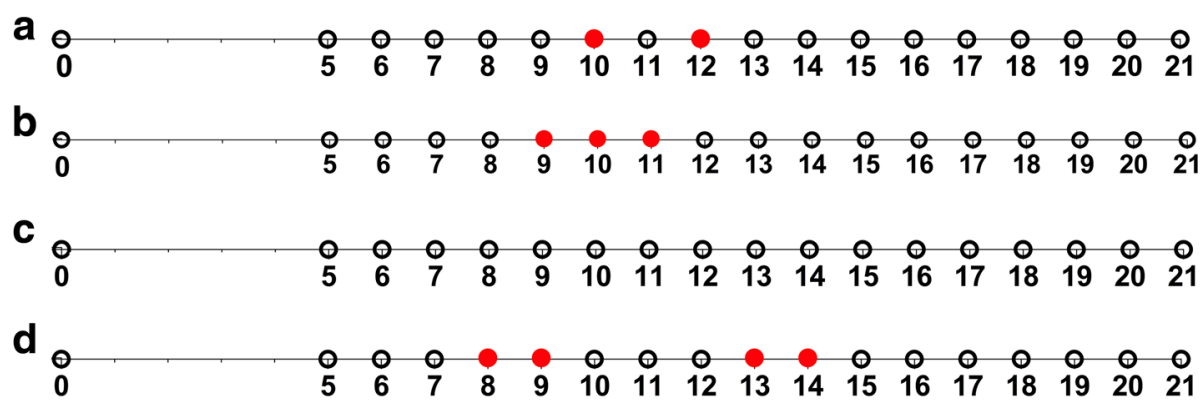

Fig. 5 Detection of T. parva DNA by p104 nested PCR in 20 tick Marikebuni calf C-1470 (a), 200 tick Marikebuni calf C-1471 (b), 20 tick Muguga calf C-1472 (c) and 200 tick Muguga calf C-1473 (d). Dates tested post-infection are represented by circles; circles are shown in red on dates where parasite DNA was detected

with the piroplasm stage are present. It is not known where or if $T$. parva sequesters in the bovine host, this study shows only that cattle remain competent reservoirs of T. parva and can infect ticks, leading to transmission, even after circulating parasitemia levels are below detection. This work confirms that these T. parva parasite stocks are tick transmissible for at least four months after infection. We have shown with this work that infections in ticks do not have to be high or detectable by currently available detection methods to transmit parasites to naïve hosts. Furthermore, transmission by ticks from these low level persistent infections resulted in sub-clinical infections, which required no treatment intervention. It follows from these results that cattle in the field that have been previously exposed to $T$. parva could serve as a source of infection for ticks, even if circulating parasites in the blood cannot be detected. Likewise, ticks feeding on cattle in endemic regions may be infective even if parasites are at undetectable levels.
Published data derived from tick feedings during acute infections suggests that the infection level in ticks is related to the parasitemia of the infected bovine host on which it feeds [35]. The sensitivity of currently available assays is not high enough to confirm that the same relationship exists in ticks fed on persistently infected cattle. Successful transmission of parasites by tick populations where parasites cannot be detected has been described for Babesia bovis [36] but has not been described for T. parva until now. We have shown here that, although individual ticks fed on persistently infected hosts have undetectable levels of infection in their salivary glands, cumulatively (in groups of 20 or more) they were able to transmit parasites to susceptible bovine hosts. Although this transmission was demonstrated in a relatively small number of cattle, it was observed in all four cattle tested ( 2 for each parasite stock), it is likely that this observation would occur in a similar fashion on a larger scale.
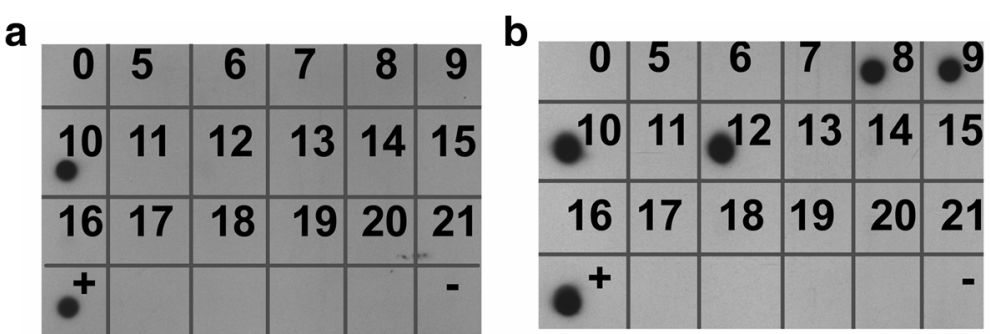

C

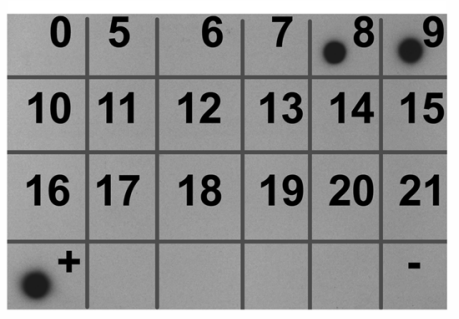

d

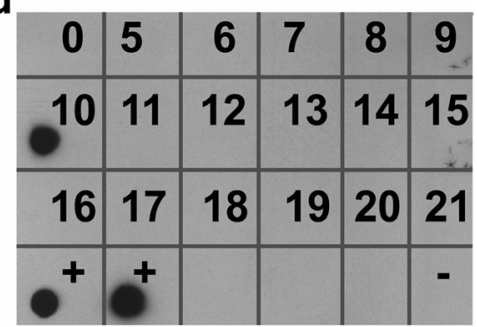

Fig. 6 Detection of T. parva DNA by 18S rRNA gene PCR and Southern blotting in 20 tick Marikebuni calf C-1470 (a), 200 tick Marikebuni calf C-1471 (b), 20 tick Muguga calf C-1472 (c) and 200 tick Muguga calf C-1473 (d) 


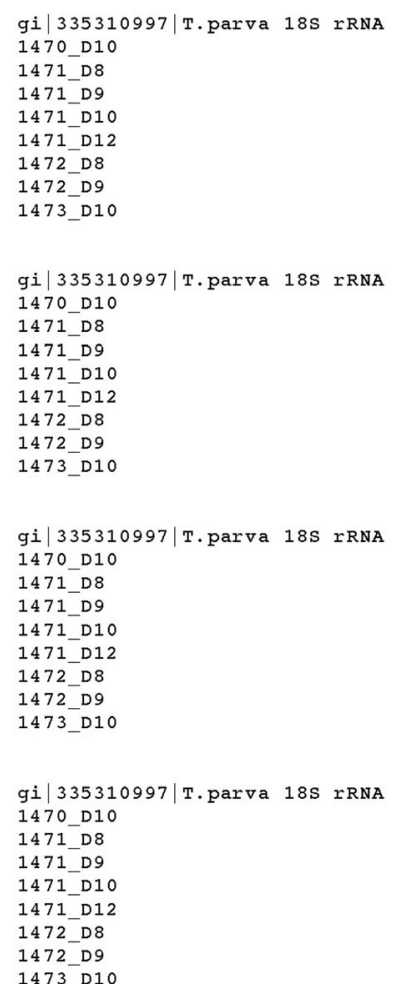

gi $335310997 \mid$ T. parva 18S rRNA

$1470 \_D 10$

1471_D8

1471_D9

1471 D10

$1471 \_\mathrm{D} 12$

1472 -D8

$1472 \_D 9$

1473_D10

gi $335310997 \mid$ T.parva $18 \mathrm{~S}$ rRNA

$1470 \_D 10$

$1470-D 10$
$1471-D 8$

1471_D8

1471_D9

1471_D10

1472_D8

1472 -D9

1473_D10

gi $|335310997|$ T.parva 18S rRNA

1470_D10

1471_D8

1471_D8

1471_D9

1471_D12

1472_D8

1472 _D9

1473_D10
TAAAGTCTTGTAATTGGAATGATGGGAATTTAAACCTCTTCCAGAGTATCAATTGGAGGG ------------A TGATGGGAATT TAAACC TC TTCCAGAGTATCAATTGGAGGG

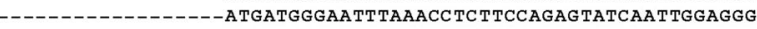
ATGATGGGAATTTAAACCTCTTCCAGAGTATCAATTGGAGGG ATGATGGGAATTTAAACCTCTTCCAGAG TATCAATTGGAGGG TTGATGGGAATTTAAACCTCTTCCAGAGTATCAADTG

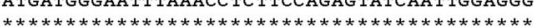

CAAGTC TGG TGCCAGCAGCCGCG TAATTCCAGC TCCAATAGCG TA TATTAAAATTGTTG CAAGTCTGGTGCCAGCAGCCGCGGTAATTCCAGCTCCAATAGCGTATATTAAAATTGTTG CAAGTCTGGTGCCAGCAGCCGCGGTAATTCCAGCTCCAATAGCG TATATTAAAATTGTTG CAAGTCTGG TGCCAGCAGCCGCG TAA TTCCAGC TCCAATAGCG TA TATTAAAATTGTTG CAAGTCTGGTGCCAGCAGCCGCGGTAATTCCAGCTCCAATAGCGTATATTAAAATTGTTG CAAG TC TGG TGCCAGCAGCCGCGGTAATTCCAGC TCCAATAGCGTATATTAAAATTGTTG CAAGTC TGG TGCCAGCAGCCGCG G TAATTCCAGC TCCAATAGCG TA TATTAAAATTGTTG CAAG TCTGG TGCCAGCAGCCGCGGTAATTCCAGCTCCAATAGCGTATATTAAAATTGTTG CAAGTCTGGTGCCAGCAGCCGCGGTAATTCCAGCTCCAATAGCGTATATTAAAATTGTTG CARETCTGGTGCCRGCAGCCGCGGTAATTCCAGCTCCAATAGCGTATATTAAATTETTG

CAGTTAAAAAGCTCGTAGTTGAATTTCTGCTGCATCGCTGTGTCCCTTCGGGGTCTCTGC CAGTTAAAAAGCTCG TAGTTGAATTTCTGCTGCATCGCTGTGTCCC TTCGGGGTCTCTGC CAGTTAAAAAGCTCG TAGTTGAATTTCTGCTGCA TCGCTGTGTCCCTTCGGGGTCTCTGC CAGTTAAAAAGC TCG TAGTTGAATTTCTGCTGCATCGCTG TGTCCC TTCGGGGTCTCTGC CAGTTAAAAAGCTCGTAGTTGAATTTCTGCTGCATCGCTGTGTCCCTTCGGGGTCTCTGC CAGTTAAAAAGCTCG TAGTTGAATTTC TGC TGCATCGCTGTGTCCC TTCGGGGTC TCTGC CAGT TAAAAAGC TCG TAGTTGAATT TC TGCTGCATCGCTGTGTCCCTTCGGGGTCTCTGC CAGTTAAAAAGCTCGTAGTTGAATTTCTGCTGCATCGCTGTGTCCCTTCGGGGTCTCTGC

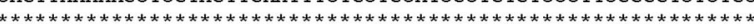

ATGTGGCTTATTTCGGACGGAGITCGCITTGICTGGATGTTTACTTTGAGAAAATTAGAG ATGTGGC TTATTTCGGACGGAGT TCGC TTTGTC TGGATGTTTACTT TGAGAAAATTAGAG ATGTGGCTTATTTCGGACGGAGTTCGCTTTGTCTGGATGTTTACTTTGAGAAAATTAGAG ATGTGGCTTATTTCGGACGGAGTTCGCTTTGTCTGGATGTTTACTTTGAGAAAATTAGAG ATGTGGCTTATTTCGGACGGAGTTCGCTTTGTC TGGATGTTTACTTTGAGAAAATTAGAG ATGTGGCTTATTTCGGACG GAGTTCGC TTTGTCTGGATGTTTACTTTGAGAAAATTAGAG ATGTGGCTTATTTCGGACGGAGTTCGCTTTGTCTGGATGTTTACTTTGAGAAAATTAGAG A TGTGGC TTATTTCGGACG GAGT TC GC TTTG TC TGGATGTTTAC TT TGAGAAAATTAGAG ATGTGGCTTATTTCGGACGGAGTTCGCTTTGTCTGGATGTTTACTTTGAGAAAATTAGAG

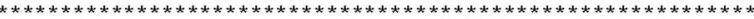

TGCTCAAAGCAGGCTTTTGCCTTGAATAGTTTAGCATGGAATAATAAAGTAGGACTTTGG TGCTCAAAGCAGGCTTTTGCCTTGAATAGTTTAGCATGGAATAATAAAGTAGGACTTTGG

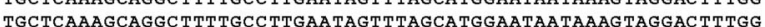
TGCTCAAAGCAGGCTTTTGCCTTGAATAGTTTAGCATGGAATAATAAAGTAGGACTTTGG TGCTCAAAGAGCTTTGCTTGAATAGTTTAGCATGGAATAATAAAGTAGGACTTTGG TGCTCAAAGCAGGCTTTTGCC TTGAATAGTTTAGCATGGAATAATAAAGTAGGACTTTGG TGCTCAAAGCAGGCTTTTGCC TTGAATAGTTTAGCATGGAATAA TAAAGTAGGACTTTGG TGCTCAAAGCAGGC TTTTGCC TTGAATAGTTTAGCATGGAATAATAAAGTAGGACTTTGG TGCTCAAAGCAGGCTTTTGCC TTGAATAGTTTAGCATGGAATAATAAAGTAGGACTTTGG

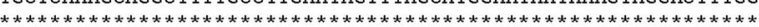

TTCTATTTTGTTGGTTTTAGG TACCAAAGTAATGGTTAATAGGAACAGTTGGGGGCATTC TTCTATTTTGTTGGTTTTAGG TACCAAAGTAATGGTTAATAGGAACAGTTGGGGGCATTC TTCTATTTTGTTGGTTTTAGGTACCAAAGTAATGGTTAATAGGAACAGTTGGGGGCATTC TTCTATTTTGTTGGTTTTAGGTACCAAAGTAATGGTTAATAGGAACAGTTGGGGGCATTC TTCTATTTTGTTGGTTTTAGG TACCAAAGTAATGGTTAATAGGAACAGTTGGGGGCATTC TTCTATTTTGTTGGTTTTAGG TACCAAAGTAATGGTTAATAGGAACAGTTGGGGGCATTC TTCTATTTTGTTGGTTTTAGG TACCAAAGTAATGGTTAATAGGAACAGTTGGGGGCATTC TTCTATTTTGTTGGTTTTAGGTACCAAAGTAATGGTTAATAGGAACAGTTGGGGGCATTC TTCTATTTTGTTGGTTTTAGG TACCAAAGTAATGGTTAATAGGAACAGTTGGGGGCATTC

GTATTTAAC TG TCAGAGGTGAAA TTCTTAGATTTGTTAAAGACGAAC TAC TGCGAAAGCA

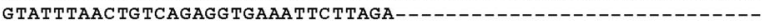

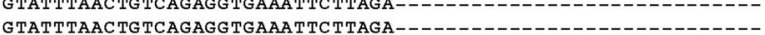
GTATTTAACTGTCAGAGGTGAAA TTCTTAGAGTATTTAACTGTCAGAGGTGTATTTAAC TGTCAGAGGT-GTATTTAACTGTCAGAGGT-

GTATTTAAC TG TCAGAGGT-GTATTTAACTG TCAGAGGT--_-_-_-_-_-

GTATTTAACTGTCAGAGGT

Fig. 7 Multiple sequence alignment for samples that tested positive by Southern blot using a T. parva 18S rRNA gene reference sequence obtained from GenBank (accession \# 335310997), priming sites for hybridization probe are underlined on the reference sequence

The ability of ticks with unapparent infection levels to transmit T. parva has far reaching implications for field surveys determining $T$. parva infection rates in both ticks and cattle. Field infection surveys of endemic areas report overall infection rates in ticks below $5 \%$ with a low level of infection in individual ticks [8, 37-39]; however, in light of the results of this study, we suggest that this grossly underestimates the number of ticks infected with $T$. parva. Likewise, single blood samples taken from cattle in T. parva endemic regions during infection surveys may underestimate infection prevalence.

Because parasite numbers associated with persistently infected cattle are at levels very close to the detection thresholds on all of our assays we investigated ways to 
increase the sensitivity in both the tick vector and bovine host. For detection of low-level infections in tick salivary glands we incubated salivary gland homogenate with primary lymphocytes. Using this method we detected one infected tick where nested p104 PCR failed to detect any (Fig. 4). This method may be useful for investigating low-level tick infection rates in field collected ticks. As the primary site of replication for T. parva in the bovine host occurs in lymphocytes, we extracted lymphocyte genomic DNA to increase detection of infection in blood. We further increased sensitivity for the p104 nested PCR by running 10 replicates for each test day, effectively increasing the volume of template tested, increasing the sensitivity by one order of magnitude. Finally, for additional confirmation of transmission, we used dot blot hybridization with chemo-luminescent detection to increase the sensitivity of our $18 \mathrm{~S}$ rRNA nPCR. Although more labor-intensive, these methods may also be employed in field surveys of $T$. parva prevalence in cattle.

Tick-based transmission models using persistently infected cattle as a source of infection may more accurately simulate the natural transmission pressures experienced by cattle in the field. Consequently, the study of transmission from persistently infected cattle may lead to a better understanding of the epidemiology of T. parva in endemic areas. Such models may also be useful as challenge models for studies testing the efficacy of future subunit vaccines [40]. In addition, our knowledge of $T$. parva immunobiology is strongly based on data collected from needle inoculation of host cattle. Needle inoculation excludes the potential impact of the tick on the route and quantity of infective pathogens introduced, eliminating the potential modulatory effects of tick salivary gland molecules on enhanced transmission and disease progression [41]. Using tick-based challenge models, infectious sporozoites are transmitted to cattle over several days and in the context of tick saliva [40, 42]. This is in sharp contrast to a one-time needle inoculation using a large number of cryopreserved sporozoites. Under conditions of endemic stability, a robust protective immune response is developed upon initial tick-borne exposure even in the absence of clinical disease. It is possible that exposure to low levels of naturally presented $T$. parva sporozoites allows the immune response to develop and act correctly, avoiding the immune dysregulation associated with severe disease [43]. Evaluation of the immune response to sporozoites naturally presented in the context of tick saliva is important to broaden our understanding of $T$. parva immunology, which has so far been founded solely on artificial needle challenge experiments.

In the broader context, a recent study observed an $89 \%$ reduction in mortality associated with $T$. parva infection in calves co-infected with less pathogenic Theileria species such as Theileria mutans and Theileria velifera [16]. Strikingly, co-infection with T. mutans and T. parva is identified to be advantageous to host growth rate compared to $T$. parva alone [44]. This suggests that the conventional concept of endemic stability may need to be expanded to include the broader community of tick-transmissible parasites [16] and uncovering the mechanism of this protection is essential. The advantage of co-infection with multiple strains of the same apicomplexan parasite is seen in malaria where infection of children with multiple Plasmodium falciparum genotypes is an indicator of lowered risk of clinical disease compared to single genotype infections [45], although once again, the mechanism remains unknown.

\section{Conclusion}

We have shown that the levels of $T$. parva parasites circulating in the blood stream that are required for transmission by $R$. appendiculatus is very low, highlighting the co-evolution of this pathogen and tick vector. We suggest utilizing a laboratory model of persistent infection as a tool to simulate and investigate hostpathogen-vector interactions occurring in endemic regions.

\section{Abbreviations}

ECF: East coast fever; ITM: Infection and treatment method; PBMC: Peripheral blood mononuclear cells

\section{Acknowledgements}

The authors would like to thank Sara Davis for her invaluable technical assistance and James Allison, Ralph Horn, Megan Blauert, Stena Erickson and Joe Hale for their monitoring and care of research animals.

\section{Funding}

This work was supported by USAID AID-BFS-P-13-00002, the Bill and Melinda Gates Foundation grant OPP1078791 and USDA, ARS, CRIS project \#5348-32000-034-00D. The funders had no role in study design, data collection and analysis, decision to publish, or preparation of the manuscript.

Availability of data and materials

All data generated or analyzed during this study are included within this published article.

\section{Authors' contributions}

The study was designed by GAS and CLO. Experiments were carried out by CLO, KM and GAS with technical assistance from staff. The manuscript was drafted by CLO and GAS. All authors read and approved the final manuscript.

\section{Ethics approval}

All experiments using animals were approved by the University of Idaho's Institutional Animal Use and Care Committee (IACUC) protocol number 2013-66. Protocols adhered to guidelines set out by the Animal Welfare Act and Animal Welfare Regulations As of Nov. 6, 2013, As found in the United States Code, Title 7 - Agriculture, Chapter 54 - Transportation, Sale, and Handling of Certain Animals, Sections 2131-2159; the Guide for the Care and Use of Agricultural Animals in Research and Teaching as well as the Guide for the Care and Use of Laboratory Animals. All animals were monitored by a registered veterinarian for symptoms of infection and general discomfort and treated accordingly. Cattle were euthanized by approved intravenous injection with sodium pentobarbital solution (Fatal Plus, Vortech Pharmaceuticals, USA). 


\section{Consent for publication}

Not applicable.

\section{Competing interests}

The authors declare that they have no competing interests.

\section{Publisher's Note}

Springer Nature remains neutral with regard to jurisdictional claims in published maps and institutional affiliations.

\section{Author details}

'Department of Veterinary Microbiology and Pathology, Washington State University, Pullman, WA, USA. ${ }^{2}$ USDA, ARS, Animal Disease Research Unit, Washington State University, Pullman, WA, USA. ${ }^{3}$ Present Address: Department of Entomology, Plant Pathology and Nematology, University of Idaho, Moscow, ID, USA

\section{Received: 27 July 2017 Accepted: 19 February 2018 Published online: 02 March 2018}

\section{References}

1. Norval RAI, Perry BD, Young AS. The epidemiology of theileriosis in Africa. Great Yarmouth: Galliard (Printers) Ltd; 1992.

2. Minjauw B, McLeod A. Tick-borne diseases and poverty. The impact of ticks and tick-borne diseases on thelivelihoods of small-scale and marginal livestock owners in India and eastern and southern Africa. UK: Research Report, DFID Animal Health Programme, Centre for Tropical Veterinary Medicine, University of Edinburgh; 2003.

3. Bishop R, Musoke A, Morzaria S, Gardner M, Nene V. Theileria: intracellular protozoan parasites of wild and domestic ruminants transmitted by ixodid ticks. Parasitology. 2004;129(Suppl. L):271-83.

4. Stagg DA, Chasey D, Young AS, Morzaria SP, Dolan $\Pi$. Synchronization of the division of Theileria macroschizonts and their mammalian host cells. Ann Trop Med Parasitol. 1980;74(2):263-5.

5. Mehlhorn H, Schein E. Redescription of Babesia equi Laveran, 1901 as Theileria equi Mehlhorn, Schein 1998. Parasitol Res. 1998;84(6):467-75.

6. Shaw MK, Tilney LG. How individual cells develop from a syncytium: merogony in Theileria parva (Apicomplexa). J Cell Sci. 1992;101(1):109-23.

7. Shiels B, Kinnaird J, McKellar S, Dickson J, Miled LB, Melrose R, et al. Disruption of synchrony between parasite growth and host cell division is a determinant of differentiation to the merozoite in Theileria annulata. J Cell Sci. 1992:101(1):99-107.

8. Young AS, Leitch BL, Newson RM, Cunningham MP. Maintenance of Theileria parva parva infection in an endemic area of Kenya. Parasitology. 1986;93(1):9-16

9. Purnell RE, Boarer CD, Pierce MA. Theileria parva: comparative infection rates of adult and nymphal Rhipicephalus appendiculatus. Parasitology. 1971;62(2): 349-53.

10. Young AS, Leitch BL, Dolan TT, Newson RM, Ngumi PN, Omwoyo PL. Transmission of Theileria parva by a population of Rhipicephalus appendiculatus under simulated natural conditions. Parasitology. 1983;86(2): 255-67.

11. Norval RA, Lawrence JA, Young AS, Perry BD, Dolan TT, Scott J. Theileria parva: influence of vector, parasite and host relationships on the epidemiology of theileriosis in southern Africa. Parasitology. 1991;102(3): 347-56.

12. Ochanda H, Young AS, Wells C, Medley GF, Perry BD. Comparison of the transmission of Theileria parva between different instars of Rhipicephalus appendiculatus. Parasitology. 1996;113(3):243-53.

13. Ochanda H, Young AS. Survival of Theileria parva-infected adult Rhipicephalus appendiculatus under laboratory and quasi-natural conditions. Exp Appl Acarol. 2003;29(3-4):355-65

14. Ochanda H, Young AS, Medley GF. Survival of Theileria parva in its nymphal tick vector, Rhipicephalus appendiculatus, under laboratory and quasi natural conditions. Parasitology. 2003;126(6):571-6.

15. Maritim AC, Young AS, Lesan AC, Ndungu SG, Mutugi JJ, Stagg DA Theilerial parasites isolated from carrier cattle after immunization with Theileria parva by the infection and treatment method. Parasitology. 1989;99(1):139-47
16. Woolhouse ME, Thumbi SM, Jennings A, Chase-Topping M, Callaby R, Kiara $\mathrm{H}$, et al. Co-infections determine patterns of mortality in a population exposed to parasite infection. Sci Adv. 2015;1(2):e1400026.

17. Coleman PG, Perry BD, Woolhouse ME. Endemic stability - a veterinary idea applied to human public health. Lancet. 2001;357(9264):1284-6.

18. Perry BD, Young AS. The past and future roles of epidemiology and economics in the control of tick-borne diseases of livestock in Africa: the case of theileriosis. Prev Vet Med. 1995;25(2):107-20.

19. Gachohi J, Skilton R, Hansen F, Ngumi P, Kitala P. Epidemiology of East Coast fever (Theileria parva infection) in Kenya: past, present and the future. Parasit Vectors. 2012:5:194

20. Di Giulio G, Lynen G, Morzaria S, Oura C, Bishop R. Live immunization against East Coast fever - current status. Trends Parasitol. 2009;25(2):85-92.

21. Patel E, Mwaura S, Kiara H, Morzaria S, Peters A, Toye P. Production and dose determination of the Infection and Treatment Method (ITM) Muguga cocktail vaccine used to control East Coast fever in cattle. Ticks Tick Borne Dis. 2016:7(2):306-14.

22. Brown CG, Radley DE, Cunningham MP, Kirimi IM, Morzaria SP, Musoke AJ. Immunization against East Coast fever (Theileria parva infection of cattle) by infection and treatment: chemoprophylaxis with N-pyrrolidinomethyl tetracycline. Tropenmed Parasitol. 1977;28(3):342-8.

23. Bishop R, Sohanpal B, Kariuki DP, Young AS, Nene V, Baylis H, et al. Detection of a carrier state in Theileria parva-infected cattle by the polymerase chain reaction. Parasitology. 1992;104(2):215-32.

24. Skilton RA, Bishop RP, Katende JM, Mwaura S, Morzaria SP. The persistence of Theileria parva infection in cattle immunized using two stocks which differ in their ability to induce a carrier state: analysis using a novel blood spot PCR assay. Parasitology. 2002;124(3):265-76.

25. Oura CA, Bishop R, Wampande EM, Lubega GW, Tait A. The persistence of component Theileria parva stocks in cattle immunized with the 'Muguga cocktail' live vaccine against East Coast fever in Uganda. Parasitology. 2004;129(1):27-42

26. Odongo DO, Sunter JD, Kiara H, Skilton RA, Bishop RP. A nested PCR assay exhibits enhanced sensitivity for detection of Theileria parva infections in bovine blood samples from carrier animals. Parasitol Res. 2010;106(2):357-65.

27. Minami T, Spooner PR, Irvin AD, Ocama JG, Dobbelaere DA, Fujinaga T. Characterisation of stocks of Theileria parva by monoclonal antibody profiles. Res Vet Sci. 1983:35(3):334-40.

28. Irvin AD, Dobbelaere DA, Mwamachi DM, Minami T, Spooner PR, Ocama JG. Immunisation against East Coast fever: correlation between monoclonal antibody profiles of Theileria parva stocks and cross immunity in vivo. Res Vet Sci. 1983;35(3):341-6.

29. Kariuki DP, Young AS, Morzaria SP, Lesan AC, Mining SK, Omwoyo P, et al Theileria parva carrier state in naturally infected and artificially immunised cattle. Trop Anim Health Prod. 1995:27(1):15-25.

30. Odongo DO, Ueti MW, Mwaura SN, Knowles DP, Bishop RP, Scoles GA. Quantification of Theileria parva in Rhipicephalus appendiculatus (Acari: Ixodidae) confirms differences in infection between selected tick strains. Med Entomol. 2009:46(4):888-94.

31. Kirstein F, Gray JS. A molecular marker for the identification of the zoonotic reservoirs of Lyme borreliosis by analysis of the blood meal in its European vector Ixodes ricinus. Appl Environ Microbiol. 1996;62(11):4060-5.

32. Kimbita EN, Silayo RS, Dolan TT. Theileria parva: in vitro studies on the effects of holding temperature, $\mathrm{pH}$ and medium on sporozoite infectivity. Trop Anim Health Prod. 2004:36(4):341-51.

33. Black WC, Piesman J. Phylogeny of hard- and soft-tick taxa (Acari:lxodida) based on mitochondrial 165 rDNA sequences. Proc Natl Acad Sci USA 1994;91(21):10034-8

34. Ueti MW, Olafson PU, Freeman JM, Johnson WC, Scoles GA. A virulent Babesia bovis strain failed to infect white-tailed deer (Odocoileus virginianus). PLoS One. 2015;10(6):e0131018.

35. Young AS, Dolan TT, Morzaria SP, Mwakima FN, Norval RA, Scott J, et al. Factors influencing infections in Rhipicephalus appendiculatus ticks fed on cattle infected with Theileria parva. Parasitology. 1996;113(3):255-66.

36. Howell JM, Ueti MW, Palmer GH, Scoles GA, Knowles DP. Persistently infected calves as reservoirs for acquisition and transovarial transmission of Babesia bovis by Rhipicephalus (Boophilus) microplus. J Clin Microbiol. 2007:45(10):3155-9.

37. Walker AR, Young AS, Leitch BL. Assessment of Theileria infections in Rhipicephalus appendiculatus ticks collected from the field. Z Parasitenkd. 1981;65(1):63-9. 
38. Ogden NH, Gwakisa P, Swai E, French NP, Fitzpatrick J, Kambarage D, Bryant M. Evaluation of PCR to detect Theileria parva in field-collected tick and bovine samples in Tanzania. Vet Parasitol. 2003;112(3):177-83.

39. Bazarusanga T, Marcotty T, Ahouandjinou AMKI, Ntumba T, Katendi C, Geysen D. Estimation of the Theileria parva entomological inoculation rate (EIR) by means of tick burden and proportion of infected questing ticks in three different farming systems in Rwanda. IJVTE. 2011;3(7):99-106.

40. Olds CL, Mwaura S, Odongo DO, Scoles GA, Bishop R, Daubenberger C. Induction of humoral immune response to multiple recombinant Rhipicephalus appendiculatus antigens and their effect on tick feeding success and pathogen transmission. Parasit Vectors. 2016;9(1):484.

41. Wikel SK. Tick modulation of host immunity: an important factor in pathogen transmission. Int J Parasitol. 1999;29(6):851-9.

42. Konnai S, Yamada S, Imamura S, Simuunza M, Chembensof M, Chota A, et al. Attachment duration required for Rhipicephalus appendiculatus to transmit Theileria parva to the host. Vector Borne Zoonotic Dis. 2007;7(2):241-8.

43. Yamada S, Konnai S, Imamura S, Simuunza M, Chembensofu M, Chota A, et al. Quantitative analysis of cytokine mRNA expression and protozoan DNA load in Theileria parva-infected cattle. J Vet Med Sci. 2009;71(1):49-54.

44. Thumbi SM, de C Bronsvoort BM, Poole EJ, Kiara H, Toye P, Ndila M, et al. Parasite co-infections show synergistic and antagonistic interactions on growth performance of East African zebu cattle under one year. Parasitology. 2013;140(14):1789-98.

45. al-Yaman F, Genton B, Reeder JC, Anders RF, Smith T, Alpers MP. Reduced risk of clinical malaria in children infected with multiple clones of Plasmodium falciparum in a highly endemic area: a prospective community study. Trans R Soc Trop Med Hyg. 1997;91(5):602-5.

\section{Submit your next manuscript to BioMed Central and we will help you at every step:}

- We accept pre-submission inquiries

- Our selector tool helps you to find the most relevant journal

- We provide round the clock customer support

- Convenient online submission

- Thorough peer review

- Inclusion in PubMed and all major indexing services

- Maximum visibility for your research

Submit your manuscript at www.biomedcentral.com/submit 